\title{
ORIGEN Y DESARROLLO DEL SISTEMA DE EDUCACIÓN TÉCNICO PROFESIONAL (ETP) PARA LA EDUCACIÓN SECUNDARIA EN ARGENTINA Y BRASIL
}

\author{
Yanina Débora Maturo \\ E-mail: yanina.maturo@unc.edu.ar \\ Instituto de Humanidades (CONICET-UNC) \\ DOI: 10.15628/rbept.2020.9821
}

Artigo submetido em: abr/2020 e aceito em: abr/2020

\section{RESUMEN}

Desde una perspectiva histórica, este artículo presenta una revisión sobre el origen y desarrollo del sistema de ETP en Argentina y Brasil. A partir de considerar los cambios en las relaciones entre Estado y sociedad se identifican diferentes períodos de desarrollo del sistema de ETP atendiendo a dos categorías teóricas: la evolución del vínculo entre trabajo y sistema de formación y, la consolidación del sistema de ETP en cada país. El análisis se basa en la revisión bibliográfica, donde se hace referencia a los marcos normativos para la ETP diseñados por cada país. Como hipótesis de trabajo se sostiene que la revisión histórica permite visualizar puntos de encuentros, tendencias, que han traspasado las fronteras ingresando como parte de una "red de políticas globales" afectando y direccionando las políticas educativas para la ETP en Argentina y Brasil; sin embargo, rasgos característicos ligados al mismo proceso de surgimiento del sistema de ETP en cada país produjeron diferencias sustanciales en el modo de reestructurar y reorganizar el sistema de ETP a lo largo de la historia y hasta la actualidad.

Palabras clave: Educación Técnico Profesional. Escuela Secundaria. Argentina. Brasil.

\section{ORIGEM E DESENVOLVIMENTO DO SISTEMA DE EDUCAÇÃO TÉCNICA PROFISSIONAL (ETP) PARA O ENSINO SECUNDÁRIO NA ARGENTINA E NO BRASIL}

\section{RESUMO}

Desde uma perspectiva histórica, este artigo apresenta uma revisão sobre a origem e o desenvolvimento do sistema ETP na Argentina e no Brasil. Ao considerar as mudanças nas relações entre Estado e sociedade, identificam-se diferentes períodos do desenvolvimento do sistema de ETP de acordo com duas categorias teóricas: a evolução da relação entre trabalho e sistema de formação e consolidação da ETP em cada país. A análise é realizada com base 
na revisão de literatura sobre o tema e os marcos regulatórios para ETP de país. Como hipótese de trabalho argumenta-se que a revisão histórica nos permite visualizar pontos de encontros, tendências que cruzaram as fronteiras, como parte de uma "rede de políticas globais" que afectam e orientam as política educacionais para o ETP na Argentina e no Brasil; no entanto, as características ligadas ao mesmo processo de surgimento do sistema de ETP em cada país produziram diferenças substanciais em como reestruturar e reorganizar 0 sistema ETP ao longo da história e na actualidade.

Palavras-chave: Educação Técnica Profissional. Escola Secundária. Argentina. Brasil.

\title{
ORIGIN AND DEVELOPMENT OF PROFESSIONAL- TECHNICAL SYSTEM (PTE) FOR SECONDARY EDUCATION IN ARGENTINA AND BRAZIL
}

\begin{abstract}
From a historical perspective, this article presents a review on the origin and development of the PTE system in Argentina and Brazil. From considering the changes in the relations between State and society, different periods of development of the PTE system are identified, considering two theoretical categories: the evolution of the link between work and the training system, and the consolidation of the secondary schools' PTE system in each country. The analysis is carried out based on the literature review, which highlights regulatory frameworks for PTE designed by each country. As a working hypothesis, it is argued that the historical review allows visualizing meeting points, trends that have crossed borders, entering as part of a "global policy network" affecting and directing educational policies for PTE in Argentina and Brazil; however, characteristic features linked to the same process of emergence of the PTE system in each country produced substantial differences in the way of restructuring and reorganizing the PTE system throughout history and until today.
\end{abstract}

Keywords: Professional-technical Education. Secondary school. Argentina. Brazil.

\section{PRESENTACIÓN}

Los cambios en los modelos de producción como en el mercado de trabajo han direccionado a lo largo de la historia las políticas educativas para el sector de la Educación Técnico Profesional (ETP), redefiniendo sus principios y funciones. El origen de las escuelas técnicas ha sido marcado por una particular vinculación con otras organizaciones, ya sean del trabajo, de la 
producción, de la ciencia y la tecnología, contribuyendo así a la reconfiguración de los escenarios sociales.

Este escrito recupera parte de una investigación ${ }^{1}$ que tuvo como propósito analizar las políticas educativas para la ETP de la escuela secundaria ${ }^{2}$ desde una perspectiva comparada entre Argentina y Brasil. En el marco de dicha investigación surgió la necesidad de partir de la reconstrucción histórica de los sistemas de ETP de cada país con el propósito de superar la lógica comparativista predominante en las últimas décadas, basada principalmente en el estudio de los fenómenos educativos como objetos aislados de su contexto, homogeneizados por medio del uso de estándares cuantitativos y estadísticos (CIAVATTA, 2000).

En este sentido, y en respuestas a las necesidades de la investigación, la decisión de incorporar una lógica histórica en el estudio de las políticas educativas para la ETP en Argentina y Brasil fue con el objetivo de preservar la singularidad de cada país en el proceso de reconstrucción de la política educativa para la ETP, sin utilizar modelos o uniformizar la recolección de los datos; con la certeza de que la construcción del sistema de ETP tanto en Argentina como en Brasil fue fruto de las dinámicas sociales, políticas y económicas de cada época que le atribuyeron un significado particular y rasgos característicos.

Para poder dar conocimiento de esos procesos se establecieron dos categorías teóricas: la evolución del vínculo entre trabajo y sistemas de formación y la conformación del sistema de ETP de la escuela secundaria en cada país. En esta línea, la reconstrucción histórica involucró la revisión de bibliografía sobre la temática y de los marcos legales que han regulado la ETP en ambos países.

El trabajo se organiza a partir de los siguientes apartados: en un primer apartado, se presentan las políticas educativas desarrolladas para organizar y administrar el sistema de ETP en cada país desde los orígenes del EstadoNación hasta la crisis del Estado Benefactor. En el segundo apartado se describen las características que asumieron las políticas educativas para el sector en el marco del impacto de las medidas neoliberales de reestructuración del Estado desarrolladas durante la década de los '90 en ambos países. En un tercer apartado se aborda el período de "recuperación" de la ETP, en donde se

\footnotetext{
${ }^{1}$ Tesis Doctoral en Ciencias de la Educación, Universidad Nacional de Córdoba (UNC), 2018.

2 Tomaremos el término "ETP de la escuela secundaria" para hablar indistintamente de lo que en Argentina se denomina "ETP de nivel medio" (Ley de Educación Técnico Profesional N ${ }^{\circ}$ 26058/2005) o "Educación secundaria de modalidad técnico profesional" (Ley Nacional de Educación № 26026/2006) y en Brasil "Educación Profesional Técnica de Nivel Medio" (Decreto 5154/2004); en tanto oferta educativa impartida en las reconocidas e históricas "escuelas técnicas".
} 
detallan cuestiones referidas a la reorganización y recuperación de la ETP en el marco de un proyecto político que ha pregonado por el crecimiento económico, el desarrollo social, la igualdad de oportunidades y la justicia social. Por último, se plantean algunas notas de cierre de lo trabajado.

\title{
2 EL SISTEMA DE EDUCACIÓN TÉCNICO PROFESIONAL EN ARGENTINA Y BRASIL: DEL ORIGEN AL ESTADO BENEFACTOR
}

\begin{abstract}
Durante el siglo XIX Argentina y Brasil emergen como naciones modernas e independientes que logran romper con los viejos vínculos coloniales. En Argentina el proceso de lucha por la independencia se inicia en 1810 culminando el 9 de julio de 1816, mientras que en el caso brasilero ocurre un poco más tarde declarándose la independencia el 7 de septiembre de 1822.

Este proceso independentista coincide con el origen de los sistemas educativos nacionales en ambos países, donde la educación tendría una función predominantemente político-cultural en la constitución del EstadoNación. Para Tedesco (2012), el período de consolidación de los sistemas educativos nacionales que va desde las últimas décadas del siglo XIX a las primeras del siglo XX, fue concebido en sus inicios como "un dispositivo de distribución social de valores y de conocimientos, según el cual cada uno debía recibir la educación requerida por el lugar que ocupaba en la estructura social" (p. 26). En este sentido, las diferencias que se pueden establecer entre los dispositivos de distribución del conocimiento de masas entre un país y otro durante esta etapa quedan expresamente ligadas a cómo cada uno fue posibilitando la adquisición de derechos a diferentes sectores de la población (MURILO DE CARVALHO, 2017).
\end{abstract}

Durante este período tanto Argentina como Brasil reconocen en la educación una capacidad redentora que permitiría sacar a la población del atraso en que se encontraba y volverla capaz de enfrentar los desafíos de la época (WEINBERG, 1995). Sin embargo, las diferencias en las trayectorias históricas de cada país llevarían a resultados distintos.

En Argentina la educación se constituiría en una estrategia independentista, una posibilidad de producir nuevos sujetos políticos capaces de imponer y mantener nuevos regímenes de gobierno. Se buscaba lograr mediante la educación la adhesión de la mayor parte de la población donde los sujetos adquirieran la categoría de ciudadano a partir de la posesión y ejercicio de ciertos derechos, todo lo cual contribuiría al ejercicio de la soberanía nacional: "el ciudadano criollo a formar presuponía la adhesión a ciertas prácticas culturales más modernas asociada a la creación de sujetos con un 
mayor nivel de individualidad y de autorregulación" (FARIA FILHO; PINEAU, 2011, p. 84).

Para Faria Filho e Pineau (2011) en Brasil, por el contrario, se observa el desarrollo de una visión más "autoritaria y excluyente" de las llamadas "clases inferiores" en las propuestas educativas de la época, que encontraría su respuesta en el origen mismo del proceso independentista:

\begin{abstract}
la independencia ocurrió un poco más tarde y fue encabezada por el príncipe heredero del trono portugués. En esta coalición las elites políticas e intelectuales brasileras, con raras excepciones, buscaron garantizar que el quiebre del pacto colonial no se hiciera con perjuicio de sus posiciones y proyectos políticos y sociales. Procuraban garantizar, sobre todo, que las ideas liberales de algunos no colisionaran con el manteniendo del régimen de la esclavitud, apoyo económico político y cultura del orden colonial que debería ser preservado en el nuevo orden Imperial. (FARIA FILHO; PINEAU, 2011, p. 92).
\end{abstract}

La función principalmente político-cultural que evidenciaría la educación en este período retardaría - tanto para el caso argentino como para el brasilero - la iniciativa de crear un sistema de "formación para el trabajo" en el marco del sistema formal de enseñanza. La propuesta de Alberdi en Argentina de "formar al productor, meter la modernización en las costumbres de la gente, imbuirlos de la fiebre de actividad y de la empresa", caducaba ante la incipiente necesidad de organización nacional y de consolidar una identidad nacional (PUIGGRÓS, 2001, p. 47). Mientras que en Brasil, la élite consideraba al trabajo manual como una actividad indigna que "predisponía al repudio en relación a las actividades artesanales y manufactureras como la carpintería, la cerrajería, el tejido, la construcción, entre otros" (PIANOVSKI VIEIRA; DE SOUZA JUNIOR, 2016, p. 154) ${ }^{3}$ por lo cual no merecía mayor interés por las políticas de Estado.

Esta dirección también se vería justificada por el rédito económico de un modelo productivo agroexportador que prevalecía en ambos países y que no exigía mayores calificaciones laborales; la formación para el trabajo no era

\footnotetext{
${ }^{3}$ Este proceso de distinción y diferenciación que surge durante la colonia esclavista y que permanece hasta la abolición de la esclavitud, va a ser determinante en lo que respecta a las representaciones sobre la noción de trabajo y las estrategias de educación que se fueron desenvolviendo a través del tiempo. (FRIGOTTO, CIAVATTA, RAMOS, 2005) (MANFREDI, 2017).
} 
un tema central de la agenda política de la época. Tal como lo expresa Garcés (2007)

\begin{abstract}
La impronta política del sistema educativo modeló una matriz cultural (...) priorizando los derechos civiles, impidiendo que se plasmaran las experiencias político-prácticas de introducción de saberes del trabajo en la escuela, resistiendo desde las utopías liberales igualitarias cualquier segmentación formal del sistema e imposibilitando un impacto troncal en el sistema educativo. (GARCÉS, 2007, p. 20).
\end{abstract}

Recién a fines del siglo XIX en Argentina toma fuerza la ETP como sistema formal de enseñanza. En 1897 el Estado Nacional crea el Departamento Industrial como anexo de la Escuela Nacional de Comercio, el cual dos años más tarde (1899) se transformaría en la primera Escuela Industrial de la Nación. ${ }^{4}$ La fundación de las primeras escuelas técnicas industriales ${ }^{5}$ durante finales del siglo XIX, conjuntamente con las Escuelas de Artes y Oficios entre 1909 y 1910, incorporaría a poblaciones en ascenso y contribuiría a cumplimentar la necesidad de formar sujetos capacitados para la industria fabril en crecimiento de comienzos del siglo XX (BOTTINELLI; SLEIMAN, 2015). Para Gallart (2006), la educación técnica en sus inicios tuvo dos objetivos manifiestos

uno, era desviar la matrícula de las modalidades mayoritarias de la educación secundaria que se consideraban "fábricas de empleo público", hacia carreras más útiles. La segunda, muy unida a la primera, era promover mandos medios para la naciente industria que se consideraba sería una actividad principal en el futuro del país. (GALLART, 2006, p. 16).

En Brasil este mismo proceso fue iniciado por Nilo Peçanha, gobernador por entonces del Estado de Río de Janeiro, quien en 1906 marcó la etapa de la enseñanza técnica oficial con la creación de cuatro escuelas

\footnotetext{
${ }^{4}$ Hoy conocida como Escuela de Educación Secundaria Técnica (EEST) № 1 "Otto Krause".

${ }^{5}$ Escuela de Oficios en la provincia de Santa Fe (1830), la Escuela de Artes y Oficios en la provincia de Entre Ríos (1868) y la Escuela de Enología en la provincia de San Juan (1862).
} 
profesionales en las ciudades de Campos, Petrópolis, Niterói y Paraíba del Sur; las tres primeras destinadas a la enseñanza de oficios y la última al aprendizaje agrícola. Luego como Presidente de Brasil, y bajo el Decreto № 7.566/1909, Peçanha creó 19 escuelas de Aprendices y Artífices en las principales capitales de la República que más tarde se transformarían en Centros Federales de Educación Profesional y Tecnológica (CEFETs) dando origen así a la Red Federal de Educación Profesional, Científica y Tecnológica. Dichas instituciones además de fortalecer la presencia del gobierno federal en los estados, tenían una finalidad moral de educar para el trabajo a los más necesitados o a los "desvalidos de la suerte" (KUENZER, 2007a, 2007b; MANFREDI, 2016).

Ahora bien, esa función político-cultural que había adquirido la educación en ambos países va a virar entrada la década de 1930 hacia una función principalmente económica en el marco del Estado Benefactor; generando las condiciones necesarias para transformar la educación en un derecho social. En Argentina, para algunos autores (GALLART, 2006; GARCÉS, 2007; PUIGGRÓS, 2001), la sociedad se estructuró así en torno al trabajo y vinculó fuertemente la escuela al mundo laboral bajo el lema del pleno empleo, impulsando la idea que la escuela brindaría la formación necesaria para la futura inserción social, política y económica de los jóvenes.

En tanto Brasil estableció en la Constitución de 1937 la obligatoriedad para la organización de escuelas de aprendices artesanos por parte de empresas y sindicatos, con el objetivo que colaboraran en la eliminación del imaginario social que las escuelas de enseñanza profesional eran solamente destinadas "a los desfavorecidos de la fortuna" o "desvalidos de la suerte": "el gobierno adoptó la enseñanza profesional, como prioridad con el fin de formar trabajadores capaces de adecuarse a la organización científica del trabajo, principio que se ajustaba a la inspiración taylorista - fordista de organización del trabajo en la producción industrial" (BELONI; WONSIK; PEREIRA, 2009, p. 4).

En Argentina, como afirma Filmus (1996) la formación del ciudadano sería reemplazada paulatinamente por la idea de formación para el trabajo:

Los nuevos roles ocupacionales exigían una alfabetización básica que el sistema educativo debía brindar (...) pero por sobre todas las cosas exigían una disciplina laboral que únicamente el sistema educativo podía brindar masivamente a millones de trabajadores (...) Estos sectores pasarían a constituir la principal fuerza laboral de las nuevas fábricas y talleres. (FILMUS, 1996, p. 24-25). 
Las transformaciones en el aparato productivo en Argentina modificarían los requerimientos educativos de la población (RAMA, 1987; WEINBERG, 1995). El creciente desarrollo industrial que caracterizó a la primera mitad del siglo XX exigió la necesidad de contar con un sistema educativo coherente capaz de fomentar la formación técnica; lo que tuvo como respuesta ${ }^{6}$ la creación de la Comisión Nacional de Aprendizaje y Orientación Profesional (CNAOP) ${ }^{7}$ el 3 de Junio de 1944 bajo la dependencia del Ministerio de Trabajo y Previsión

La CNAOP quiso instaurar una enseñanza técnica postbásica que complementara la expansión de la educación primaria en el primer gobierno peronista (1946). Impartía una educación vocacional técnica focalizada en las ascendentes capas obreras y respondía a un modelo industrializador. En lugar de la preponderancia de alumnos de clase media común en la escuela industrial, en las escuelas de la CNAOP predominaba el origen social popular. (GALLART, 2006, p. 19).

La CNAOP estaba principalmente compuesta por las escuelas fábrica de nivel secundario y por la Universidad Obrera Nacional ${ }^{8}$, en estas instituciones no sólo se impartían los contenidos académicos tradicionales sino que también se impartían saberes sobre derecho laboral y sindical (PINEAU, 1997). Junto a la Dirección General de la Enseñanza Técnica -la cual agrupaba a las Escuelas Industriales de la Nación, las Escuelas para Niñas y las Misiones Monotécnicas de Cultura Rural y Doméstica y organizaba la oferta de las Escuelas de Artes y Oficios y las Escuelas Profesionales, etc.-, la CNAOP significó una respuesta satisfactoria para articular bajo un modelo de institución

\footnotetext{
${ }^{6}$ Para Filmus (1996), recién durante el gobierno peronista se pudo evidenciar una respuesta concreta no sólo a la demanda del sistema productivo sino a la necesidad de una integración social genuina, pues como afirma el mismo autor: "los intentos de restauración oligárquica ocurridos en la década, los '30 mostraron la incapacidad de los sectores tradicionales para restablecer la hegemonía de un sistema basado en la exclusión de las nuevas mayorías que emergieron junto con la industrialización." (FILMUS, 1996, p. 23).

7 Al culminar el gobierno de Perón (1955), "las escuelas técnicas sumando industriales y CNAOP, contaban con más de 86.000 matriculados, en comparación con sólo 34.000 en 1945 . Si bien esto fue parte de una gran expansión de la educación secundaria, el porcentaje de matriculados en la educación técnica sobre el total de media creció de 13,3 a 18,3\%" (GALLART, 2006, p. 20).
}

${ }^{8}$ Actualmente Universidad Tecnológica Nacional (UTN). 
escolar una nueva manera de concebir la relación educación-trabajo que requería la época.

Ahora bien, a partir de la caída de Perón esta concepción "económicocentrista" de la ETP se ve profundizada. Entre 1955 y 1973 se sientan las bases de una Argentina moderna (Estado desarrollista) y la educación técnica tendrá un papel sustancial en lo que respecta a la formación de un ciudadano capaz de generar progreso y producir un cambio social. Los gobiernos pos-peronistas buscan dar respuesta a la demanda incipiente del sector industrial, pero marcando cierta diferencia con las propuestas de sus antecesores (PINEAU, 1997): el 15 de noviembre de 1959 se crea el CONET. Un organismo colegiado y autárquico perteneciente al Ministerio de Educación y Justicia ${ }^{9}$, financiado a través de un impuesto a la nómina del empleo industrial y que estaba compuesto por una representación tripartita de empresarios, sindicatos y el Estado. A través del mismo, se procuraron nuevos criterios tendientes a orientar la capacitación técnica y profesional acorde a las características económicas, sociales y de formación profesional que requería la época.

A través de la creación de la Escuela Nacional de Educación Técnica (ENET) el CONET tuvo el propósito de unificar las escuelas técnicas en un modelo propio y único que apoyara el esfuerzo industrializador. Estas escuelas agruparían a partir de 1965 las antiguas escuelas técnicas industriales, las de la CNAOP y las escuelas de artes y oficios. Todas ellas tuvieron desde ese momento una organización similar y un plan común dividido en distintas especialidades

\footnotetext{
${ }^{9}$ Las funciones del CONET eran: a) Darse su propio reglamento y el de sus dependencias directas; b) Elevar al Ministerio de Educación para su aprobación los proyectos de planes de estudio y los programas respectivos ajustados a dichos planes; c) Designar, remover, trasladar y sancionar a su personal técnico, directivo, docente y administrativo, con sujeción a las normas establecidas por el Estatuto del Docente y por el Estatuto para el Personal Civil de la Nación; d) Fijar los deberes y atribuciones del personal técnico, directivo, docente y administrativo y estatuir todo lo referente a las tareas de los institutos a su cargo; e) Fijar los derechos de exámenes, matrículas y otros análogos; f) Proyectar su presupuesto anual de gastos y manejar los fondos asignados por la ley de lo que dará cuenta en la forma establecida por las disposiciones legales en vigencia; g) Disponer sobre la construcción, refección y conservación de edificios educacionales, así como adquisición de material didáctico, y todo cuanto concurra a los fines especificados en el enunciado de este artículo; h) Estudiar los libros de textos y elevar dictamen al Ministerio de Educación y Justicia para su aprobación; i) Reglamentar las condiciones de ingreso del alumnado, correlación de estudios, sistemas de clasificaciones, exámenes, promociones y la revalidación de certificados; establecer un sistema de becas a otorgarse a estudiantes de todo el país; j) Otorgar los respectivos certificados de estudios, elevando al Ministerio de Educación los legajos de los graduados para el otorgamiento de los títulos y, habilitación profesional; k) Ejercer el contralor de los establecimientos adscriptos; I) Reglamentar el funcionamiento de cooperadoras, asociaciones de ex alumnos y egresados.
} 
Los estudios postprimarios comenzaban con tres años comunes a la educación secundaria (ciclo básico), más el agregado de horas de taller en contraturno, complementado por un ciclo de tres años de especialización. Las especialidades más numerosas continuaron siendo Mecánica, Electricidad, Construcción y algo menor, Química. Se crearon nuevas especialidades dirigidas a otras ramas de la industria, pero se respetó el esquema común señalado. El currículo que seguía a grandes rasgos el modelo de la escuela industrial, estuvo en vigencia hasta el fin del siglo XX, y sólo fue incorporando nuevas especialidades y adecuaciones tecnológicas (GALLART, 2006, p. 20).

Las ENET conjuntamente con las Escuelas Técnicas Provinciales ${ }^{10}$, conformaron la oferta de ETP de la escuela secundaria del país, perdurando esta distinción hasta iniciada la década de 1990, momento donde la ETP en Argentina sufre el más profundo "desguace" de su historia en manos de la Reforma Educativa iniciada por el menemismo (MATURO \& RUBIO, 2008).

En lo que respecta a Brasil la creación del Ministerio de Educación y Salud Pública en 1937, durante la etapa del "Estado Nuevo", fue crucial para el desarrollo de la ETP. Dentro de la órbita del Ministerio de Educación y Salud Pública se creó la Inspectoría de Enseñanza Técnico Profesional que se encargó de la supervisión de las Escuelas de Aprendices Artesanos, hasta entonces ligadas al Ministerio de Agricultura. Se inició así un período de significativa expansión, marcado por una política de creación de nuevas escuelas industriales y la introducción de nuevas especializaciones en las escuelas existentes, en donde prevalecieron tanto iniciativas públicas como privadas (MANFREDI, 2016; PIANOVSKI VIEIRA; de SOUZA JUNIOR, 2016).

Sin embargo, y a diferencia de Argentina, en 1942 surge el "Sistema S". Un sistema paralelo al sistema público conformado por el Servicio Nacional de Aprendizaje Industrial (SENAI), que en 1946 se vería acompañado por la creación del Servicio Nacional de Aprendizaje Comercial (SENAC), el Servicio Social del Comercio (SESC) y el Servicio Social de la Industria (SESI).

El "Sistema S" se constituyó como una red de educación profesional de carácter privado, pero de alcance nacional, organizado y gerenciado por órganos sindicales de representación empresarial y por las confederaciones y federaciones empresariales. Durante el período cívico-militar (1964-1985) el

\footnotetext{
${ }^{10}$ Cabe destacar, tal como lo expresa Gallart (2006), que la demanda de ETP durante el Estado desarrollista fue tan importante que las provincias comenzaron a crear sus propias escuelas técnicas; sin embargo, las mismas "fueron consideradas de baja calidad" en relación a las ya consolidadas ENET.
} 
"Sistema S" fue en uno de los principales referentes de formación para el trabajo (MANFREDI, 2016), característica que perdura hasta nuestros días ${ }^{11}$.

Asimismo, este período estuvo caracterizado en Brasil por una marcada lógica dualista entre trabajo manual y trabajo intelectual, que promovía la misma diferenciación por clases sociales: la primera destinada a la elite y la segunda a las clases bajas. Si bien la sanción de la Ley de Directrices y Bases en 1961 flexibilizó el traspaso de una modalidad a otra, la dualidad persistió

Los mecanismos legales y las estructuras formativas históricamente construidas, a lo largo de la década de 1940 a 1970, fueron cristalizando concepciones y prácticas escolares dualistas: de un lado la concepción de educación escolar académico-generalista, en la cual los alumnos tenían a un conjunto básico que eran cada vez más amplios a medida que proseguían sus estudios y de otro, la Educación Profesional, en la cual según Alves (1997) el alumno recibía un conjunto de informaciones relevantes para el dominio de su oficio, sin profundización teórica, científico y humanista, que le brinde condiciones de proseguir estudios 0 de calificar para otros dominios. (MANFREDI, 2017, p. 56).

Durante los gobiernos militares brasileros (1964 a 1985) se desarrollaron grandes proyectos de desarrollo nacional, ligados básicamente a la explotación del petróleo y los productos agropecuarios que demandaron mano de obra calificada. La perspectiva tecnicista que incorporaron los proyectos benefició sobre todo al sector privado de la educación profesional, el cual a la vez se vio beneficiado por un programa denominado Programa Intensivo de Mano de Obra que concedía incentivos fiscales a las empresas para que ellas mismas lleven a cabo la capacitación de sus obreros.

\section{LA DÉCADA DE LOS NOVENTA: LAS POLÍTICAS NEOLIBERALES Y LA DESARTICULACIÓN DEL SISTEMA DE EDUCACIÓN TÉCNICO PROFESIONAL}

\footnotetext{
11 En la actualidad también pertenecen a este grupo el Servicio de Apoyo a la Pequeña y Mediana Empresa (SEBRAE) y el Servicio Social de las Cooperativas de Prestación de Servicios (SESCOOP).
} 
Este período se caracteriza por un proceso de profundos cambios en el panorama internacional que modifica las condiciones sociales, económicas, culturales y políticas de los países Latinoamericanos, poniendo restricciones a sus acciones en todos los ámbitos, incluyendo el educativo. Argentina y Brasil llevan a cabo una fuerte reestructuración del Estado a través de procesos de privatización, descentralización y desregulación como resultado de las recomendaciones de Organismos Internacionales de Crédito (OIC) y en el marco de las negociaciones por la deuda externa. Tal como afirma Berchansky (2008, p 81) "el ajuste estructural de la profunda crisis del Estado en los dos países, generó un clima propicio para el desarrollo de tendencias minimalistas sobre el Estado".

Respectivamente, en el plano económico, ambos países apostaron por lograr la estabilidad económica a partir de la creación del Plan de Convertibilidad y el Plan Real, lo que permitió contener el proceso inflacionario que aquejaba a ambos países desde décadas anteriores. Con ello iniciarían un conjunto de reformas del Estado en busca de su racionalización y modernización a través de procesos de privatización de empresas estatales, cambios en la forma de gestión de las políticas públicas y la desregulación de la administración pública federal por medio de la descentralización (FILMUS, 1996; OLIVEIRA, 2011).

En el plano social, se podían vislumbrar los efectos de la globalización a través de las demandas de mayor calificación vinculadas a las transformaciones en la ciencia y tecnología y a la competitividad en el mercado internacional. Se manifiestan en este contexto argumentaciones económicas para el desarrollo de políticas para la ETP que impregnan de racionalidad instrumental las estrategias que expresaba el discurso político para la modalidad (ALMANDOZ, 2010).

Los modos de regulación de las políticas educativas que caracterizan a los procesos de reforma se fundamentan en valores y criterios que proceden del campo de la economía y que alteran la relación que ésta mantiene con el ámbito educativo. Se produce una dependencia del ámbito educativo en relación a una lógica económica a través de la implantación de esquemas de mercado que tiene que ver con

la aplicación de procesos de evaluación y acreditación, descentralización y mayor autonomía de las escuelas y los docentes en relación con el trabajo pedagógico, la administración y el financiamiento, procedimientos de gestión y administración escolar orientados a criterios de eficiencia y productividad inspirados en modelos gerenciales y adecuación de los currículos a las 
necesidades de formación de los recursos humanos del sector productivo. (ALMANDOZ, 2000, p. 10)

La política de Estado reemplaza los esquemas centralistas para involucrar cada vez más a los grupos y organizaciones en la cuestión social y educativa con el propósito de reubicar los mecanismos de poder y control; para gestionar los conflictos que pudieran suceder y para aumentar la desgastada legitimidad de la administración estatal (CARRANZA et al., 1999). En este nuevo esquema administrativo, las escuelas asumen un papel importante en tanto logran mayor autonomía para incidir en la vida de sus propios actores y en las decisiones institucionales, curriculares y organizativas.

Las transformaciones que sufre el Sistema Educativo Argentino durante la década de los noventa (bajo la presidencia de Carlos Menen 1989 1999) se pueden ver plasmadas en: la sanción de la Ley 24.049 de Transferencia de los Servicios Educativos en 1.991, la Ley Federal de Educación 24.195 en 1.993, la Ley de Educación Superior 24.521 en 1.995 y la Reforma Constitucional en 1994 que introduce nuevas disposiciones para el sector educativo ${ }^{12}$. Los principios e intenciones que guiaron el proceso de reforma educativa se justificaron en la necesidad de lograr consolidar la identidad e integración nacional, la democratización de la educación, la igualdad de oportunidades y de posibilidades, la calidad de la enseñanza, la equidad y la justicia social, así como también la democratización y fortalecimiento de la democracia y la valorización del trabajo y el aporte de la educación al desarrollo regional y nacional (CARRANZA et al., 1999; MIRANDA; SENÉN GONZÁLEZ; LAMFRI, 2003).

En Brasil, a mediados de esa misma década, el Presidente Fernando Henrique Cardozo (1995-2002) también promovía amplias transformaciones en el ámbito educativo. De acuerdo a Oliveira (2011)

\footnotetext{
12 De acuerdo a Miranda, Senén González e Lamfri (2003), entre los aspectos principales que configuran la Transformación Educativa en el marco de la Ley Federal de Educación, podemos destacar: una nueva estructura para el Sistema Educativo Nacional, nuevas responsabilidades para el Estado Nacional y las provincias en lo que respecta al gobierno y administración de la educación, la evaluación de la calidad de la educación como estrategia para fijar y controlar el cumplimiento de la política educativa y de garantizar el cumplimiento de los principios, objetivos y funciones del Sistema Educativo Nacional, la transformación curricular a través de tres escalas de trabajo (confección de los Contenidos Básicos Comunes, de los Diseños Curriculares Jurisdiccionales y Proyecto Curricular Institucional), la formación, actualización y capacitación docente, los nuevos lineamientos para el financiamiento de la educación y la formulación de políticas compensatorias para acompañar todo este proceso de transformación.
} 
El conjunto de reformas implantadas en la educación brasileña en ese período resultó en la reestructuración de la educación escolar en sus aspectos relativos a la organización, a la redacción de los currículos por medio de los Parámetros Curriculares Nacionales (PCN), a la evaluación por el incremento al Sistema Nacional de Evaluación de la Educación Básica (SAEB) y luego el Examen Nacional de Cursos (ENC), a la gestión y su financiamiento, mejor ejemplificado por la creación del Fondo de Mantenimiento y Desarrollo de la Enseñanza Fundamental y de la valorización del Magisterio (FUNDEF). Especialmente en la educación básica, los cambios realizados redefinirán su estructura. Las alteraciones en la legislación educativa brasileña consumaron esa nueva reconfiguración, teniendo como expresión mayor la Ley de Directrices y Bases de la Educación Nacional Nº394 /1996. (OLIVEIRA, 2011, p. 326).

La Ley de Directrices y Bases № 9493/1996 y el Decreto Federal № 2208/1997 serían los instrumentos legales más importantes en la transformación de la educación secundaria en general y de la ETP en particular, obligando a las instituciones a adecuarse a tales prescripciones.

En lo que respecta a Argentina, la sanción de la Ley Federal de Educación № 24195/1993 y la consecuente implementación de un nuevo currículum en la educación secundaria o "Polimodal" (como lo denominó dicha ley) generaron cambios sustanciales en la organización y gestión institucional de las escuelas técnicas, llevando casi a su extinción en varias provincias.

La Ley Federal de Educación propuso una Educación General Básica de nueve años de duración y estructurada en tres ciclos de tres años cada uno y, una Educación Polimodal de tres años de duración, ésta última ofreciendo diferentes "modalidades" en las cuales se desestimaba la ETP. En vistas a ofrecer un espacio de formación profesional para los alumnos, se ofreció un "Trayecto Técnico Profesional" bajo el formato de "contraturno" y de carácter "optativo" que pretendió en su momento sustituir la formación brindada por las tradicionales escuelas técnicas. El impacto social que generó la omisión de la educación técnica en los textos legales se trató de emendar a través de estos "Trayectos Técnicos Profesionales" ofrecidos en el marco de la educación polimodal. Como afirma Gallart (2006)

Los cambios en la enseñanza, su articulación con los cambios tecnológicos, y la crisis de la demanda laboral en el sector industrial también dejaron su huella en la educación técnica. Los cambios 
educativos de la década del noventa, y en particular la descentralización, el incremento de la matrícula y la Reforma Educativa, estuvieron cerca de firmar el certificado de defunción de la escuela técnica. (Gallart, 2006, p.79)

Bajo la perspectiva de un modelo económico que privilegiaba el desarrollo del sector de bienes y servicios se produce un proceso de desarticulación de la educación técnica en Argentina que se materializa en la transferencia de las ENET a la gestión provincial, la desaparición del CONET y la consecuente creación del Instituto Nacional de Educación Tecnológica (INET) en 1995. Este último tuvo por objeto dotar al Ministerio de Educación de un instrumento ágil para el desarrollo de las políticas relacionadas con la ETP.

Mientras tanto, en Brasil, la reforma de la enseñanza secundaria común como la de la ETP tuvo como justificativo "la mejora de la oferta educativa y su adecuación a la nuevas demandas económicas y sociales de la sociedad de la globalización, portadora de nuevos patrones de productividad y competitividad" (MANFREDI, 2011, p. 71). La escuela secundaria, similar al caso argentino, se configuró bajo un solo modelo en donde se trató de articular conocimientos, competencias para la ciudadanía y para el mundo del trabajo sin ser específicamente profesionalizante. En este sentido

La educación profesional será desenvuelta en articulación con la educación regular o en modalidades que contemplen estrategias de educación continua, pudiéndose realizar en escuelas de enseñanza regular, en instituciones especializadas o en los ambientes de trabajo (MANFREDI, 2011, p. 72)

El Decreto Federal № 2208 de 1997, que reglamentó el Inciso 2o del Art. 36 de los Art. 39 a 42 de la Ley de Directrices y Bases No 9394/1996, establece modificaciones en la oferta de ETP produciendo profundas transformaciones organizativas y curriculares. De acuerdo al Art. 3 del mismo Decreto, la ETP comprenderá los siguientes niveles:

I. básico: destinado a la calificación, recalificación y reposición de trabajadores, independientemente de la escolaridad previa;

II. técnico: destinado a proporcionar habilitación profesional a alumnos matriculados o egresados de la enseñanza media, debiendo ser impartido en la forma establecida por éste Decreto; 
III.tecnológico: correspondiente a cursos de nivel superior en el área tecnológica, destinados a egresados de la enseñanza media y técnica.

En este sentido, el nivel técnico se circunscribe al nivel de la escuela secundaria y se destina a los matriculados y/o egresados del nivel secundario. Con una organización y currículum en módulos, donde cada módulo se constituía en una unidad de contenidos que podían brindar una habilitación específica para el mundo del trabajo, esta nueva forma de organización de la ETP de la escuela secundaria se diferenciaba claramente de la escuela secundaria común y se ofrecía bajo dos opciones: concomitante o consecuente. La primera habilitando al alumno a cursar conjuntamente la escuela secundaria común con algún Curso Técnico (módulos) y la segunda, habilitando al alumno a cursar una vez concluida la escuela secundaria común. Para Koritiake (2008), la puesta en acto del Decreto 2208/1997 implicó:

[...] desvinculación pedagógica e institucional de la enseñanza técnica de la enseñanza media común, modularización de los cursos, reformulación de los currículos por competencias en el aprendizaje a aprender, introducción de componentes curriculares profesionalizante en la enseñanza media, introducción de componentes curriculares de formación general en la enseñanza técnica, reformulación de los modelos convencionales de adquisición de conocimiento, sistematización de mecanismos de evaluación de la calidad de la enseñanza. La Teoría del Capital Humano y el ideario neoliberal como eje de su reformulación de la enseñanza técnica y media. (KORITIAKE, 2008, p 164-171).

De esta manera, la transformación de la ETP en ambos países consistió en la introducción de modificaciones en la organización y estructura curricular de la ETP de la escuela secundaria a partir de la creación de un sistema paralelo de enseñanza que ofrecía una formación específica de acuerdo a la orientación. En el caso de Argentina este discurso se materializó en la creación de los "Trayectos Técnicos Profesionales" (GALLART, 2006) y en Brasil en la configuración de una "Enseñanza Técnica Modular" (ZIBAS, 2007). Las reformas en el currículum estuvieron orientadas a proponer un conjunto amplio de competencias generales consideradas como transversales al nivel de enseñanza y de acuerdo a los requerimientos del mercado (JACINTO, 2010). 
Las medidas adoptadas por ambos países durante este período llevaron casi a la desaparición de la tradicional escuela técnica y la desestructuración de todo el sistema de enseñanza técnico profesional tradicional (FERRETI, 2009; GALLART, 2006). Se produce así una dependencia en relación a una lógica económica a través de la implantación de "esquemas de mercado" basado en la formación de competencias (ALMANDOZ, 2000). En palabras de Garcés (2007)

\begin{abstract}
En su esquema simple, el neoliberalismo planteará que si los individuos en su paso por la escuela no adquieren saberes que sean útiles a sus intereses (en realidad identificados con los intereses del capital y no con los intereses sociales) habrá que sustituirlos por 'competencias', pretendiendo así otorgar un nuevo sentido, con la marca del eficienticismo que pregona. (GARCÉS, 2007, p. 47)
\end{abstract}

Estas transformaciones producen además una clara evidencia de un desplazamiento del papel legitimador del Estado en favor del mercado, quien será el encargado de determinar las "competencias" que deben brindarse en la escuela para lograr el éxito en el mundo del trabajo y determinarán la currícula de la escuela secundaria en general.

\title{
3 LA POLÍTICA EDUCATIVA PARA LA EDUCACIÓN TÉCNICO PROFESIONAL EN ARGENTINA Y BRASIL A PARTIR DEL NUEVO MILENIO $^{13}$
}

Un cambio significativo en el plano político promediando la primera década del siglo XXI va a potenciar la posibilidad de recuperar las históricas escuelas técnicas. El ascenso de Néstor Kirchner a la presidencia en Argentina y de Luiz Inácio Lula Da Silva en Brasil, si bien no implicó la ruptura total con las políticas neoliberales que caracterizaron la década de los '90, constituyó una diferenciación significativa respecto a la etapa anterior (BERCHANSKY, 2008; FELDFEBER; GLUZ, 2011).

Durante el año 2001 Argentina transita uno de los períodos de crisis económica, financiera e institucional más profundos, que paradójicamente se

${ }^{13}$ Este apartado recupera parte de un trabajo publicado. (MATURO, 2016). 
constituye en la antesala para la toma de decisiones que se desarrollarán desde el gobierno electo en el 2003. Al período de crisis sucede un período de crecimiento sostenido y actividad económica que posibilita pensar en una educación en prospectiva, que claramente se enmarca en principios como la justicia social, la independencia económica y la soberanía política, con una sólida presencia del Estado en la apuesta al mercado interno y a la reindustrialización del país. Como afirma Garcés (2007)

\begin{abstract}
aunque las ideas que pregonan el caos como fuente de las transformaciones sociales no parecen tener asidero científico, no puede dejar de advertirse, que, a ésta, la mayor crisis de la historia argentina reciente, ha sucedido un período de bonanza económica y significativa - aunque insuficiente - mejora de la situación social, tan inédita como la crisis que la precediera. (GARCÉS, 2007, p. 13)
\end{abstract}

Se inicia un nuevo período para la ETP y una reconfiguración de la articulación educación-trabajo en base a un nuevo modelo de relación Estadosociedad-sistema productivo. Las políticas educativas formuladas postulan una nueva forma de pensar la relación entre sistema educativo y sistema productivo en Argentina, reposicionando la función de la ETP y de la escuela técnica:

Un conjunto de factores presentes al promediar la primera década del siglo contribuye a potenciar esta oportunidad [...] el reconocimiento, desde el Estado, de la Educación Técnica como parte fundamental del sistema educativo, y en consecuencia las acciones de apoyo que se desprenden de la Ley recientemente promulgada y que buscan la mejora de la calidad de la Educación Técnica Profesional [...]. (GALLART, 2006, p. 80)

Durante el transcurso de la presidencia de Néstor Kirchner se sanciona la Ley de Educación Técnico Profesional (ETP) № 26058/2005. Un nuevo marco normativo para la ETP que intenta por un lado, atender los problemas heredados de la "aplicación dispar" de la Ley Federal de Educación y "el bache legal" que había dejado la Reforma Educativa de los '90 en torno a la educación técnica (FELDFEBER; GLUZ, 2011; FINNEGAN; PAGANO, 2007; GALLART, 2006; MIRANDA, 2012; SENÉN GONZÁLEZ, 2008). Por otro lado, aspira a organizar el servicio educativo de la formación técnica en sus diferentes niveles (medio y superior no universitario), modalidades (formal y no formal) y la 
formación profesional. Así la ley se constituye en una de las principales normas que integran las bases legales que regulan el derecho a la educación en la República Argentina.

En lo que respecta a Brasil, desde inicios de la Presidencia de Lula da Silva se intentó recuperar el papel del Estado como impulsor y promotor de las políticas socio-económicas en las cuales, al igual que en el caso argentino, la ETP pasaría a tener un papel relevante. En palabras de Manfredi (2016)

\begin{abstract}
En el discurso, las políticas públicas en el campo de la educación profesional y tecnológica se pensarán en otra lógica: de la educación profesional como un derecho social, por tanto, deber del Estado; la construcción de mecanismos de participación y control social; el esfuerzo de aproximar las políticas educativas con las políticas de desarrollo socio-económico direccionadas para la reducción de la exclusión económica y social. (MANFREDI, 2016, p.189).
\end{abstract}

En este sentido, una de las primeras medidas adoptadas fue la sanción del Decreto $N^{\circ} 5154 / 2004$, el cual introdujo importantes modificaciones en el sistema educativo de la ETP reglamentando el Art. 2 de la LBD/1996. Allí se establecieron los niveles para la ETP y se posibilitó la integración curricular de la ETP a la escuela secundaria común, que había sido desarticulada en los '90 por medio del Decreto $N^{\circ} 2208 / 1997$.

La organización y administración del sistema de ETP también se vería favorecido por la posterior sanción de la Ley № 11741/2008 que redimensiona, institucionaliza e integra las acciones de la formación técnico profesional a la escuela secundaria; y la Ley $\mathrm{N}^{\circ} 11892 / 2008$, de establecimiento de la Red Federal de Educación Profesional y Tecnológica bajo la dependencia del sistema de educación federal junto con la creación de los Institutos Federales de Educación Profesional, Ciencia y Tecnología, como mecanismos de expansión tanto de la Red Federal como de las redes estaduales y municipales. Como estrategias de expansión de la ETP durante este período se destacan principalmente durante los dos gobiernos de Lula da Silva, los Programas "PROEJA" (2005), "Brasil Profesionalizante" (2007) y "Educación Media Innovadora" (2009); y durante el gobierno de Dilma Rousseff, el "Programa de acceso a la educación media y el empleo (PRONATEC)" (2012).

En su contenido, los nuevos marcos normativos en Argentina y Brasil buscan diferenciarse de las orientaciones profesionalizantes de corte neoliberal que se sostuvieron durante los años '90, fundadas básicamente en el 
desarrollo de competencias profesionales para puestos de trabajo específicos. En términos generales, las definiciones sobre la ETP en ambos países tendieron a finalidades formativas de carácter más integral, vinculadas al crecimiento personal, laboral y social, la formación ciudadana, humanística y científico-tecnológica.

Podemos destacar cambios sustanciales en el sistema de ETP de cada país, a partir del análisis de las siguientes dimensiones: gobierno y administración; estructura y organización, y mecanismos de financiamiento.

\subsection{GOBIERNO Y ADMINISTRACIÓN}

En Argentina, la Ley de ETP ${ }^{14}$ es el marco normativo por excelencia para regular y ordenar la ETP de nivel medio, superior no universitario y la formación profesional en todo el territorio. El Estado Nacional a través del Poder Ejecutivo Nacional, conjuntamente con los Poderes Ejecutivos de las provincias y del Gobierno de la Ciudad Autónoma de Buenos Aires, toman la centralidad del gobierno y administración de todo el sistema educativo, incluyendo la ETP; con el deber de garantizar la educación gratuita y obligatoria a todos los habitantes del territorio argentino en igualdad de calidad, oportunidades y de manera equitativa desde los 4 a los 17 años de edad. ${ }^{15}$

EI INET se constituye en el principal organismo del Ministerio de Educación de la Nación que tiene como función la coordinación de la puesta en acto de las políticas para la ETP. Este organismo cuenta con dos ámbitos de consulta y acuerdo permanente: la Comisión Federal de Educación Técnico Profesional (CFETP) y el Consejo Nacional de Educación Trabajo y Producción (CoNETyP). Conjuntamente realizan las propuestas de ofertas formativas, de gestión de fondos (inversión) y de mejoramiento de la calidad educativa para presentar al Consejo Federal de Educación para su posterior aprobación. Asimimo, la Ley de ETP puso en marcha tres instrumentos estratégicos para la regulación y ordenamiento del sistema de ETP: la homologación de títulos y

\footnotetext{
${ }^{14}$ En el año 2006 se sanciona la Ley Nacional de Educación № 26026 reforzando el valor de la Ley de ETP al establecer que la ETP se regirá por las disposiciones de la Ley de ETP y reconociéndola como "la modalidad" de la Educación Secundaria y la Educación Superior responsable de la formación de técnicos medios y técnicos superiores en áreas ocupacionales específicas y de formación profesional (Art. 38, Capítulo VI, Título II).

15 Por medio de la Ley № 27.045 de diciembre de 2014 se estable como obligatoria la sala de 4 años y se modifica el Art. 16 de la Ley de Educación Nacional № 26026/2006 que establecía la obligatoriedad escolar desde los cinco años. Asimismo, se modifican los Art. 18 y 19 para establecer el nivel inicial como una unidad pedagógica desde los 45 días a los 5 años, siendo obligatorios los dos últimos.
} 
certificaciones, el Catálogo Nacional de Títulos y Certificaciones y el Registro Federal de Instituciones de ETP.

En lo que respecta a Brasil, la Unión, el Distrito Federal, los estados y municipios tienen el deber de garantizar la educación gratuita y obligatoria de manera organizada a través de un régimen de colaboración entre los diferentes sistemas de enseñanza para los ciudadanos de 4 a 17 años de edad. ${ }^{16}$ Si bien la Unión tiene la coordinación de la política educativa nacional, los diferentes sistemas de enseñanza estadual y municipal también tendrán libertad de organización en los términos que establece la LDB (Art. 8 y sus incisos).

La Unión se encarga de elaborar el Plan Nacional de Educación, organizar, mantener y desarrollar los órganos e instituciones del sistema educativo oficial federal, proporcionar asistencia técnica y financiera a los Estados, Distrito Federal y municipios, y colabora con los mismos para la elaboración de las bases curriculares de la educación obligatoria con el objetivo de asegurar una formación básica común (Directrices Curriculares Nacionales). El Consejo Nacional de Educación, como parte de la estructura educativa y bajo la dependencia del Ministerio de Educación de la Nación, cumple las funciones de regulación y supervisión del sistema educativo en coordinación con la Unión.

El organismo que planifica, orienta, coordina y evalúa el proceso de formulación y desarrollo de la política educativa para la ETP en todo el territorio brasilero es la Secretaría de Educación Profesional y Tecnológica (SETEC) y al igual que en Argentina cuenta con un Sistema Nacional de Información de la Educación Profesional y Tecnológica (SISTEC) y un Catálogo Nacional de Cursos Técnicos. que permiten mensualmente, previo registro de las instituciones acreditadas, informar sobre la oferta de cursos de ETP de la escuela secundaria.

\subsection{ESTRUCTURA Y ORGANIZACIÓN}

La Ley de ETP en Argentina articula la educación formal con la no formal y la formación general con la profesional, en el marco de una concepción de educación continua y permanente. Para ello diferencia los fines, objetivos y propósitos de la ETP de la escuela secundaria y superior no universitaria -que se ofrecerá en las históricas escuelas técnicas y en instituciones de ETP de nivel superior no universitario respectivamente-, de los fines, objetivos y propósitos de la formación profesional que se llevará a cabo en las distintas

\footnotetext{
${ }^{16}$ La extensión de la obligatoriedad y la gratuidad de la educación básica en Brasil queda determinada por Ley $N^{\circ} 12796$ del 4 de abril de 2013.
} 
instituciones de formación profesional (centros de formación profesional, escuelas de capacitación laboral, centros de educación agraria, misiones monotécnicas, escuelas de artes y oficios, escuelas de adultos con formación profesional, o equivalentes). (Ley de ETP, Art. 9)

Para la estructuración y organización de la modalidad de ETP de la escuela secundaria, como se observa en el Figura 1, la Ley de Educación Nacional № 26206/2006 brinda la posibilidad a cada jurisdicción de optar entre dos alternativas de estructura para los niveles de la educación primaria y secundaria: a) una estructura de seis años para el nivel de la educación primaria y de seis años para el nivel de la educación secundaria $0, b)$ una estructura de siete años para el nivel de la educación primaria y de cinco años para el nivel de la educación secundaria.

FIGURA 1: Cuadro La ETP de la escuela secundaria en el Sistema Educativo Argentino - LEN N² 26026/2006

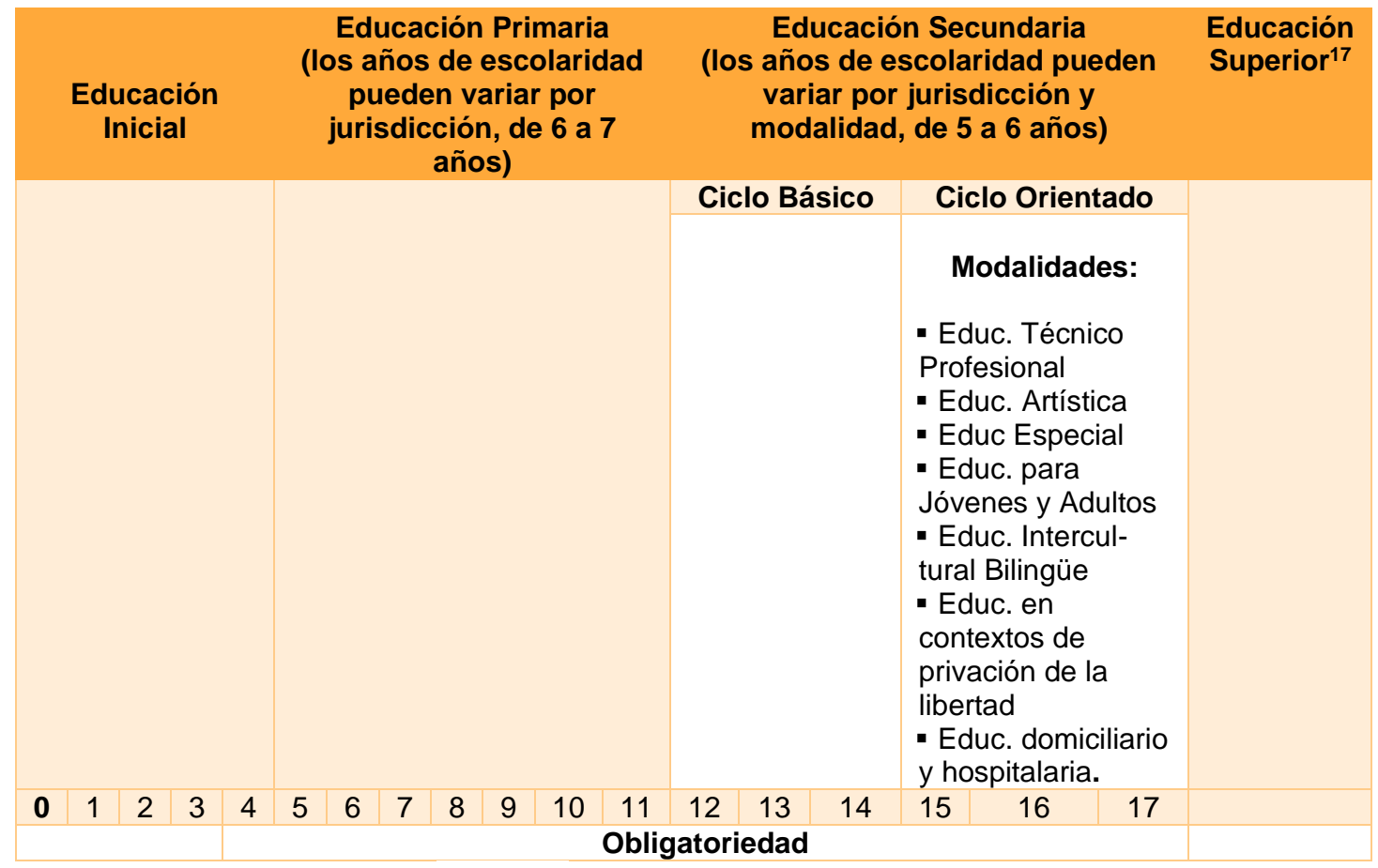

Fuente: MATURO (2014, p. 105)

\footnotetext{
17 La ETP de nivel superior se regirá por las disposiciones de la Ley de Educación Superior № 24521/1995 y su articulación con el nivel medio quedará supeditado a las acciones desarrolladas por cada jurisdicción.
} 
Con respecto a la estructura y organización de la ETP en Brasil la Ley $\mathrm{N}^{\circ} 11741 / 2008$ establece que la preparación para el mundo del trabajo y su consecuente habilitación profesional podrán ser ofrecidas en los mismos establecimientos de enseñanza media o en cooperación con instituciones especializadas en ETP. Los diplomas obtenidos en la modalidad de la ETP de nivel medio, siempre y cuando estén registrados en el SISTEC, habilitarán profesionalmente y también para el cursado de estudios superiores.

En la Ley 11741/2008 también se hace referencia a que la ETP integra diferentes niveles y modalidades de la educación, organizando los cursos por ejes tecnológicos y posibilitando de esta manera la construcción de diferentes itinerarios formativos: formación inicial y continua o de cualificación profesional; ETP de nivel medio, en sus modalidades articulada o subsecuente y ETP de graduación o pos-graduación. Tal como se lo puede ver en el Figura 2.

FIGURA 2: La ETP de la escuela secundaria en el Sistema Educativo Brasilero LDB/1996, Decreto 5154/2004 y Ley No 11741/2008.

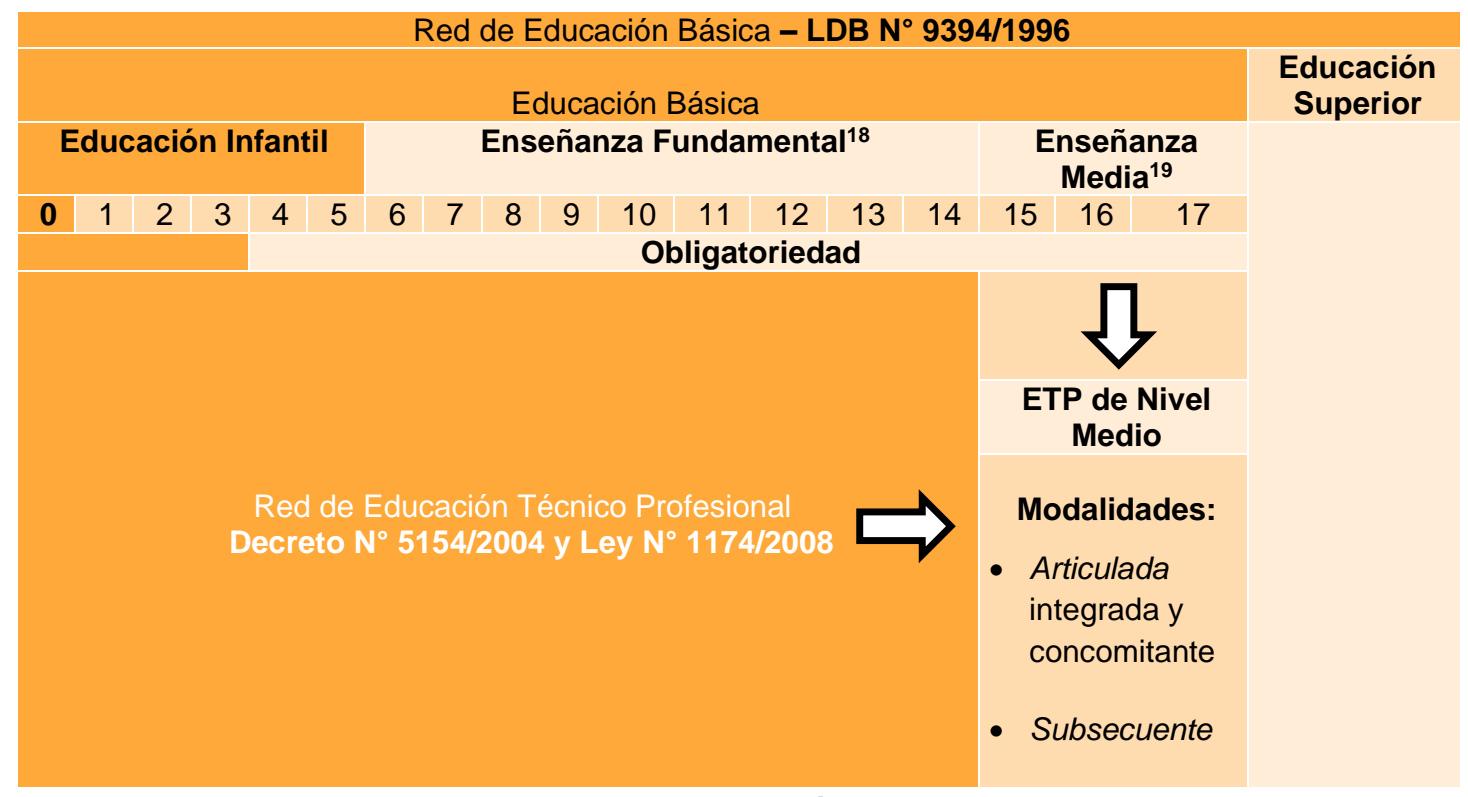

Fuente: elaboración propia

En lo que respecta específicamente a la oferta de ETP de la escuela secundaria en Brasil, esta adquiere dos modalidades: articulada, en tanto

${ }^{18}$ Equivalente a parte de la Educación Primaria en Argentina.

${ }^{19}$ Equivalente a la Educación Secundaria en Argentina. 
enseñanza técnica integrada a la media (ETIM) y concomitante; y subsecuente, como cursos posteriores dirigidos a personas que han terminado la educación secundaria.

De acuerdo a esta organización, la ETP articulada puede ofertarse de dos maneras: integrada, ofrecida sólo a aquellos que han concluido la educación primaria con el objetivo de que el alumno obtenga un título habilitante de formación técnica y profesional en la misma institución y bajo una matrícula única (inscripción), o concomitante, es decir para quienes ingresen a la escuela media o ya estén cursando la misma generando matrículas distintas (inscripciones) para cada curso y pudiendo desarrollarse en la misma institución, en diferente institución o en instituciones educativas que tengan acuerdos de intercomplementariedad para una propuesta educativa unificada. (Art. 36, incisos del A al D, LDB/9394; modificados por Ley 11741/2008).

\subsection{FINANCIAMIENTO}

El financiamiento de la educación en Argentina está regulado por la Ley de Financiamiento Educativo № 26075/2005. Para Miranda (2012) esta ley recupera la preocupación por los recursos para educación fijando "mayores precisiones" sobre las fuentes reales de financiamiento, el destino de los fondos, los mecanismos de cumplimiento de las metas y los criterios de redistribución de recursos.

En el texto de la ley se expone la voluntad del gobierno nacional, de las provincias y la Ciudad Autónoma de Buenos Aires de aumentar progresivamente el presupuesto para educación, ciencia y tecnología hasta alcanzar en el año 2010 el 6\% en el Producto Bruto Interno (PBI). Respecto a la modalidad de la ETP, en el Art. 2, inciso h, ratifica entre sus propósitos: "Fortalecer la educación técnica y la formación profesional impulsando su modernización y vinculación con la producción y el trabajo. Incrementar la inversión en infraestructura y equipamiento de las escuelas y centros de formación profesional".

Además de los fondos provenientes de la Ley de Financiamiento Educativo vía Ministerio de Educación, Ciencia y Tecnología, la Ley de ETP crea por medio del Art. 52 el "Fondo Nacional para la Educación Técnico Profesional" el cual 
será financiado con un monto anual que no podrá ser inferior al CERO COMA DOS POR CIENTO $(0,2 \%)$ del total de los Ingresos Corrientes previstos en el Presupuesto Anual Consolidado para el Sector Público Nacional, que se computarán en forma adicional a los recursos que el Ministerio de Educación, Ciencia y Tecnología tiene asignados a otros programas de inversión en escuelas. Este Fondo podrá incorporar aportes de personas físicas y jurídicas, así como de otras fuentes de financiamiento de origen nacional o internacional.

La creación de un fondo presupuestario propio para la ETP refuerza el compromiso del Estado Nacional por mejorar de manera continua las condiciones materiales de las instituciones de ETP a través de optimizar la calidad de los aprendizajes de los alumnos con mejor infraestructura y más equipamiento para la enseñanza práctica. El fondo se distribuye a través de tres vías que pretenden el desarrollo de políticas específicas: los Programas Federales, los Planes de Mejora Jurisdiccionales y los Planes de Mejora Institucionales.

En lo que respecta a Brasil, el financiamiento de la educación en general se realiza a través de las recaudaciones de los impuestos propios de la Unión, los Estados, el Distrito Federal y los municipios; como así también de las transferencias constitucionales y de otro tipo, de las recaudaciones de la asignación por escolaridad y otras atribuciones sociales, de la recaudación de impuestos y de otros recursos previstos por la ley (Art. 68, LDB 9394/1996). Esos fondos son utilizados también para el desarrollo y sostenimiento de la ETP

La Unión aplicará, anualmente, nunca menos de dieciocho, y los Estados, el Distrito Federal y los municipios, el veinticinco por ciento, o lo que consta en las respectivas Constituciones o Leyes Orgánicas, de los ingresos resultantes de impuestos, comprendidas las transferencias constitucionales, en el mantenimiento y desarrollo de la enseñanza pública. (Art. 69, LDB 9394/1996).

Cabe destacar que el sistema de ETP también se ve beneficiado por la sanción de la Ley $\mathrm{N}^{\circ} 12858 / 2013$, en septiembre de 2013, la cual fija un plan para subir el gasto educativo del $6 \%$ al $10 \%$ del PIB para el año 2022. Dicha ley establece que el $75 \%$ de las regalías por concepto de la explotación petrolera se destine a educación y otro $25 \%$ al sector salud. Asimismo, 
establece que se direccione para el sector educativo el $50 \%$ del Fondo Social Pre-Sal y se aplique el $50 \%$ de las recaudaciones recibidas por el Fondo Social de la Unión hasta que se cumplan las metas establecidas por el Plan Nacional Educativo (PNE) 2011-2020.

\section{NOTAS CONCLUSIVAS}

Una lectura retrospectiva nos permite dar cuenta de los avatares que ha sufrido la ETP en la historia de cada país, en relación al lugar que le han otorgado determinados modelos políticos, económicos y productivos. Asimismo, nos permitió visualizar puntos de encuentros, tendencias, que han traspasado las fronteras y han ingresado como parte de lo que autores como Ball (en AVELAR, 2016) denominan una "red de políticas globales", para hablar de la diseminación de políticas en el mundo. Ahora bien, el mismo proceso de recontextualización de esas políticas -conjugadas con las características históricas y contextuales de cada país- es lo que ha generado diferencias entre Argentina y Brasil, afectando la manera en que se ha desenvuelto la reestructuración y reorganización del sistema de ETP a lo largo de la historia.

En ambos países la ETP tuvo un crecimiento sostenido sin mayores transformaciones hasta inicios de la década de los '90, cuando producto de las políticas neoliberales se introducen modificaciones en la organización y estructura curricular de la ETP que deriva en la creación de un sistema paralelo de enseñanza. Las medidas adoptadas por ambos países durante este período llevaron casi a la desaparición de la tradicional escuela técnica y a la desestructuración de todo el sistema de enseñanza técnico profesional tradicional (FERRETI, 2009; GALLART, 2006).

El ascenso de Néstor Kirchner a la presidencia en Argentina y de Lula Da Silva en Brasil, si bien no implicó la ruptura total con las políticas neoliberales que caracterizaron la década de los '90 -las cuales impulsaron el achicamiento de las funciones del Estado en materia educativa a través de la privatización y la descentralización de servicios-, constituyeron una diferenciación respecto a la etapa anterior (FELDFEBER \& GLUZ, 2011). A través de enmiendas o de la creación de nuevas normas, ambos países devuelven la denominación de modalidad a la ETP dentro de la estructura de los sistemas de enseñanza y la posicionan como una estrategia fundamental para el desarrollo y crecimiento económico.

En ese contexto son notables los cambios introducidos en la estructura del sistema de ETP en Brasil a partir de la incorporación de los Institutos Federales. Estas instituciones no solo aparecen ampliando la oferta de ETIM sino que incorporan un nuevo formato de "institución total"; ofreciendo todos 
los niveles de la educación formal con orientación técnico profesional y también con propuestas de carácter no formal. En Argentina, si bien se da todo un proceso de recuperación de la ETP de la escuela secundaria, éste conserva un formato institucional muy ligado al viejo modelo de escuela técnica del CONET, lo que da cuenta de cierta imposibilidad para modificar estructuras tradicionales de funcionamiento y gestión. Asimismo, en términos generales podemos destacar que las políticas para la ETP en Argentina y Brasil presentan grandes avances respecto a las décadas anteriores; ya sea en materia de financiamiento (posibilitando mejoras en las escuelas existentes o creando nuevas, potenciando las condiciones de enseñanza-aprendizaje) o a través de programas que acompañaron la trayectoria escolar de los alumnos para su egreso de la modalidad, etc.

Si bien los efectos/resultados de la implementación de las políticas diseñadas durante los gobiernos de Kirchner y Lula Da Silva son desarrollados en otros trabajos, como posibles líneas de investigación a futuro, queda por interrogarnos por las políticas educativas para la ETP que se vienen desarrollando en ambos países a partir del 2015. El cambio de gobierno en Argentina y Brasil pareciera estar contribuyendo a una restauración del orden neoliberal de la mano de nuevas políticas públicas que afectan a diferentes ámbitos de la sociedad, incluyendo el educativo (FILMUS, 2017; GENTILI, 2016). Pareciera que Argentina y Brasil están destinados nuevamente a compartir parte de una historia.

\section{REFERÊNCIAS}

ALMANDOZ, María Rosa. Políticas para la educación técnico profesional en la Argentina. In: ALMANDOZ, María Rosa (Org.). Educación y trabajo: articulaciones y políticas. Buenos Aires: IIPE - UNESCO, 2010, p. 233-274.

ALMANDOZ, María Rosa. Sistema Educativo Argentino. Escenarios y Políticas. Buenos Aires: Santillana, 2000.

AVELAR, María (2016). Interview with Stephen J. Ball: Analysing his contribution to education policy research. Education Policy Analysis Archives, 24(0), 24. https://doi.org/10.14507/epaa.24.2368

BELONI, Belmiro; WONSIK, Ester; PEREIRA, Arcelo. Contexto histórico e políticas públicas da educação profissional no século XXI: apontamentos introdutórios. In: Campo Mourão: [s.n.], 2009. Disponível em:

http://www.fecilcam.br/nupem/index.php?option=com-content\&task=view\&id= 15\&ltemid=14>. 
BERCHANSKY, Jorge. Século XXI: novo imperialismo e educação. Brasil Argentina nos governos Lula e Kirchner. Educação superior e a reforma. Teses Doutorado, Faculdade de Educação -Universidade Estadual do Campinas, Brasil, 2008.

BOTTINELLI, Leandro; SLEIMAN, Cecilia. La educación técnica en la Argentina. Observatorio Educativo de la UNIPE, p. 1-9, 2015. (Dossier).

CARRANZA, Alicia; KRAVETZ, Silvia; CASTRO, Alejandra; et al. Descentralización, autonomía, y participación. Páginas. Revista de la Escuela de Ciencias de la Educación - UNC, v. 1, 1999. (1). Disponível em: $\leq$ https://revistas.unc.edu.ar/index.php/pgn/article/view/14966>. Acesso em: 8 set. 2018.

CIAVATTA, Maria. Quando nós somos o outro: questões teóricometodológicas sobre os estudos comparados. Educação \& Sociedade, v. 21, n. 72, p. 197-230, 2000.

FARIA FILHO, Luciano; PINEAU, Pablo. La educación y la cuestión de la construcción de identidades modernas en el siglo XIX. Los casos de Argentina y Brasil. In: VIDAL, Diana; ASCOLANI, Adrián (Orgs.). Reformas Educativas en Brasil y Argentina. Ensayos de historia comparada (18202000). Buenos Aires: Biblos, 2011, p. 81-104.

FELDFEBER, Myriam; GLUZ, Nora. Las políticas educativas en Argentina: herencias de los '90, contradicciones y tendencias de "nuevo signo".

Educação \& Sociedade, v. 32, n. 115, p. 339-356, 2011.

FERRETI, Celso. A reforma do ensino técnico da década de 1990: entre a proposta e a prática. Revista Brasileira de Estudos Pedagógicos, v. 90, p. 11-31, 2009. (224).

FILMUS, Daniel. Educar para el mercado. Escuela, Universidad y ciencia en tiempos de neoliberalismo. Buenos Aires: Octubre, 2017.

FILMUS, Daniel. Estado, sociedad y educación en la Argentina de fin de siglo: proceso y desafíos. Buenos Aires: Troquel, 1996.

FILMUS, Daniel. La explosión de la educación técnica. Disponível em: $\leq$ http://editorial.unipe.edu.ar/la-educacion-en-debate/numeros-29-al-37-ano20 15/item/36-la-explosion-de-la-educacion-tecnica>. Acesso em: 29 out. 2018.

FINNEGAN, Florencia; PAGANO, Ana. El derecho a la educación en

Argentina. Buenos Aires: Fundación Laboratorio de Políticas Públicas., 2007. 
GALLART, María Antonia. La escuela técnica industrial en Argentina: ¿un modelo para armar? Montevideo: CINTERFOR-OIT, 2006.

GARCÉS, Luis. La formación para el trabajo en el sistema educativo. Una mirada histórica. In: GARCÉS, Luis (Org.). ¿De la escuela al trabajo?: la educación y el futuro laboral de los jóvenes en tiempos de globalización. Buenos Aires: Ediciones del Signo, 2007, p. 13-42. (Colección Razón Política/Educación).

GENTILI, Pablo. Golpe en Brasil: genealogía de una farsa. Buenos Aires: CLACSO, 2016.

INET. Institucional. Instituto Nacional de Educación Tecnológica. Disponível em: shttp://www.inet.edu.ar/index.php/institucional/historia/>.

JACINTO, Claudia. Reformulaciones recientes acerca de la formación para el trabajo en la educación secundaria general. In: TENTI FANFANI, Emilio (Org.). Educación y Trabajo. Interrelaciones y Políticas. Buenos Aires: IIPE UNESCO, 2010, p. 111-135.

KORITIAKE, Luiz Antonio. Reestruturação produtiva e educação: um estudo sobre a proposta do Centro Estadual de Educação Tecnológica Paula Souza para o ensino médio e técnico. Tese Doutorado, Universidade Metodista de Piracicaba, Piracicaba, 2008.

KUENZER, Acacia. Da dualidade assumida à dualidade negada: 0 discurso da flexibilização justifica a inclusão excludente. Educação \& Sociedade, v. 28, p. 1153-1178, 2007. (100).

KUENZER, Acacia. Ensino médio: construindo uma proposta para os que vivem do trabalho. Cortez: São Paulo, 2007.

MANFREDI, Silvia. Educação profissional no Brasil: atores e cenários ao longo da história. São Paulo: Paco Editorial, 2016.

MATURO, Yanina. La Educación Técnico Profesional de nivel medio en Argentina y Brasil: una lectura del marco normativo vigente. In: Revista Latinoamericana de Educación Comparada (RELEC), Sociedad Argentina de Estudios Comparados, № 10, Año 7, p. 66-80, 2016.

MATURO, Yanina. La educación técnica en Argentina: de la "reforma educativa"-década de los 1990-a la ley de educación técnico profesional.

Revista Exitus, V. 4, p. 95-109, 2014.

MATURO, Yanina \& RUBIO, Amalia. La transformación educativa en las ex escuelas técnicas de Córdoba (1996 - 2005): una mirada desde el currículum. Tesis de Licenciatura, Universidad Nacional de Córdoba, Córdoba, 2008. 
MIRANDA, Estela. La gran apuesta: Ley de Financiamiento de la Educación y Ley de Educación Técnico Profesional. 2012.

MIRANDA, Estela; SENÉN GONZÁLEZ, Silvia; LAMFRI, Nora. Políticas de Reforma del Sistema Educativo en los noventa. Nuevas configuraciones emergentes a partir de la Ley Federal de Educación y su implementación en Córdoba. Córdoba: Editorial Brujas, 2003.

MURILO DE CARVALHO, José. Cidadania no Brasil. O longo caminho. Río de Janeiro: Civilizacao Brasileira, 2017.

OLIVEIRA, Dalila Andrade. Das políticas de governo à política de Estado: reflexões sobre a atual agenda educacional brasileira. Educação \& Sociedade, v. 32, p. 323-337, 2011. (115).

PIANOVSKI VIEIRA, Alboni; DE SOUZA JUNIOR, Antonio. A educação profissional no Brasil. Revista Interacções, v. 12, p. 152-169, 2016. (40).

PINEAU, Pablo. La vergüenza de haber sido y el dolor de ya no ser: los avatares de la educación técnica entre 1955 y 1983. In: PUIGGRÓS, Adriana (Org.). Historia de la Educación Argentina. Buenos Aires: Galerna, 1997, v. VIII Dictaduras y utopías en la historia reciente de la educación argentina, $p$. 379-402.

PUIGGRÓS, Adriana. ¿Qué pasó en la educación argentina? Buenos Aires: Galerna, 2001.

RAMA, Gerardo. Educación y sociedad en América Latina. OEA. [s.I.: s.n.], 1987.

SENÉN GONZÁLEZ, Silvia. Políticas, leyes y educación. Entre la regulación y los desafíos de la macro y la micropolítica. In: PERAZZA, Roxana (Org.).

Pensar en lo público. Notas sobre Educación y Estado. Buenos Aires: Aique, 2008, p. 81-118.

TEDESCO, Juan Carlos. Educación y justicia social en América Latina. Buenos Aires: Fondo de Cultura Económica, 2012.

WEINBERG, Gregorio. Modelos educativos en la historia de América Latina. Buenos Aires: AZ Editora, 1995.

ZIBAS, Daniel. Una visión general de la educación técnica en Brasil. La legislación, las críticas, los impases y los avances. In: Argentina: [s.n.], 2007. 